\title{
Maximal volume representations are fuchsian
}

\author{
Stefano Francaviglia* \\ University of Pisa, Italy \\ e-mail: francavi@sns.it
}

\author{
Ben Klaff ${ }^{\dagger}$ \\ University of Texas, Austin \\ e-mail: klaff@math.utexas.edu
}

November 2, 2004

\begin{abstract}
We prove a volume-rigidity theorem for fuchsian representations of fundamental groups of hyperbolic $k$-manifolds into Isom $\left(\mathbb{H}^{n}\right)$. Namely, we show that if $M$ is a complete hyperbolic $k$-manifold with finite volume, then the volume of any representation of $\pi_{1}(M)$ into $\operatorname{Isom}\left(\mathbb{H}^{n}\right)$, $3 \leq k \leq n$, is less than the volume of $M$, and the volume is maximal if and only if the representation is discrete, faithful and "k-fuchsian".
\end{abstract}

\section{Introduction}

The main result of this paper is a generalization and streamlined proof of a result which is often referred to as the "representation volume rigidity" theorem:

Theorem 1.1. Let $M$ be an oriented, connected, complete, real hyperbolic $k$-manifold of finite volume, with $k \geq 3$. Let $\rho: \pi_{1}(M) \rightarrow \operatorname{Isom}\left(\mathbb{H}^{n}\right)$ be a representation of its fundamental group into the group of isometries of hyperbolic n-space. Then the volume of $\rho$ is less than or equal to the volume of $M$, and equality holds if and only if $\rho$ is $k$-fuchsian, i.e., a discrete and faithful representation into the group of isometries of a $k$-dimensional subspace of $\mathbb{H}^{n}$.

*The first author was supported by an INdAM fellowship and the Department of Applied Mathematics of the University of Pisa.

$\dagger$ The second author was also supported by a CIRGET fellowship and by the Chaire de Recherche du Canada en algèbre, combinatoire et informatique mathématique de l'UQAM. 
In the case $k=n=3$ and $M$ is closed, this result was proved, following original ideas of W. Goldman, M. Gromov, and W. Thurston [10, 12, by N. Dunfield 6 . The result was extended to the case when $M$ has finite volume by the authors [8, 11].

The new ingredient in the present proof is the use of natural maps, or the barycenter method, a technique introduced and developed by G. Besson, G. Courtois, and S. Gallot. (See, for example, [2, 3, 4.) A key step in the proof is the first author's generalization of the B-C-G method so as to be able to construct natural maps for representations [7.

We remark that in addition to the new proof offered here, the original "volume-rigidity of representations" result itself has been generalized, in that it deals with the case when the target dimension is greater than that of the domain. We also note that Besson, Courtois and Gallot have recently obtained a similar result [5].

The paper is organized as follows. In Section 2, we give the necessary background and definitions, including the definition of the volume of a representation. In Section 3, we give the proof of Theorem 1.1] in the case $M$ is closed. Finally, in Section 4, we complete the proof of Theorem 1.1, giving the proof for non-compact, complete, finite-volume manifolds.

ACKnowledgements. The authors warmly thank Juan Souto for the stimulating conversations about the B-C-G techniques while in Cambridge and in Pisa. The second author would also like to thank Pete Storm, Benson Farb, Steve Boyer, and Stephan Tillmann, as well as the Universita di Pisa for their hospitality.

\section{Definitions and notation}

Throughout Sections 2 and 3, $M$ will denote a closed, oriented hyperbolic $k$ manifold, $k \geq 3$. We suppress a choice of basepoint in $M$ and let $\Gamma=\pi_{1}(M)$ denote the fundamental group of $M$. We let $\rho: \Gamma \rightarrow \operatorname{Isom}\left(\mathbb{H}^{n}\right)$ denote a representation of $\Gamma$ into the group of isometries of $\mathbb{H}^{n}$.

By a pseudo-developing map for $\rho$, we mean a piecewise smooth map $D=D_{\rho}: \widetilde{M} \rightarrow \mathbb{H}^{n}$ which is $\rho$-equivariant, i.e., such that

$$
D(\gamma \cdot x)=\rho(\gamma) \cdot D(x)
$$

for every $x \in \widetilde{M}=\mathbb{H}^{k}$, the universal cover of $M$, and for every $\gamma \in \Gamma$. 
Given any representation $\rho$, one can construct a pseudo-developing map for $\rho$ as follows: lift a smooth triangulation for $M$ to $\widehat{M}$, and then recursively define the map $D$ on the $i$-skeleta, $0 \leq i \leq k$, by choosing images for a complete system of orbit representatives for the $i$ th skeleta, and then extending the map equivariantly.

We now introduce the notion of the volume of a representation. Let $h$ denote the hyperbolic metric on the target $\mathbb{H}^{n}$ and let $D$ be a pseudo-developing map for $\rho$. The pullback $D^{*} h$ of $h$ along $D$ is a (possibly degenerate) pseudometric on $\widetilde{M}$ and hence induces a $k$-form $\tilde{\omega}_{D}=\left|\operatorname{det} D^{*} h\right|$ on $\widetilde{M}$. Since the map $D$ is $\rho$-equivariant, $D^{*} h$ and hence $\tilde{\omega}_{D}$ are $\Gamma$-invariant. Hence the $k$-form $\tilde{\omega}_{D}$ descends to a $k$-form $\omega_{D}$ on $M$.

Definition 2.1 (Volume of a pseudo-developing map). The volume $\operatorname{vol}(D)$ of a pseudo-developing map $D$ for a representation $\rho$ is defined by

$$
\operatorname{vol}(D)=\int_{M} \omega_{D}
$$

We can now make the following

Definition 2.2 (Volume of a representation). The volume $\operatorname{vol}(\rho)$ of a representation $\rho$ is defined by

$$
\operatorname{vol}(\rho)=\inf _{D}\{\operatorname{vol}(D)\},
$$

where the infimum is taken over the set of all pseudo-developing maps $D$ for $\rho$.

Note that $\operatorname{vol}(D)$ and hence $\operatorname{vol}(\rho)$ are non-negative real numbers. Also, note that $\operatorname{vol}(D)$ is not invariant under $\rho$-equivariant homotopy. Hence the volumes of two pseudo-developing maps for a given representation can be different. We use the above definition of representation-volume in order to deal with the case $n \neq k$. (Compare the definition of representation-volume and the consequent property of invariance under homotopy in 66, 8.) Finally, we point out that in the non-compact case, the definition of volume of a representation involves another condition. (See Section 4.)

\section{The compact case}

When $M$ is compact, the proof of Theorem 1.1 goes as follows. First, we invoke an existence result due to the first author [7], which says that there is 
a pseudo-developing map $F$ for $\rho$ such that $\operatorname{vol}(F) \leq \operatorname{vol}(M)$. The inequality then follows by the definition of $\operatorname{vol}(\rho)$.

Next, we use the hypothesis that $\operatorname{vol}(\rho)=\operatorname{vol}(M)$, some elementary Riemannian geometry, and the properties of the pseudo-developing map $F$ to conclude that $F$ is a Riemannian isometry from $\mathbb{H}^{k}$ to a $k$-dimensional hyperbolic subspace of $\mathbb{H}^{n}$. (This then reduces the remainder of the proof to the case $k=n$.) It is then easy to conclude that $F$ is a covering map onto its image, and it follows that $\rho$ is discrete and faithful.

Finally, we show the (easier) converse, namely that if $\rho$ is a discrete, faithful representation into the group of isometries of a $k$-dimensional hyperbolic subspace of $\mathbb{H}^{n}$, then $\operatorname{vol}(\rho)=\operatorname{vol}(M)$.

The following result is proved in [7].

Lemma 3.1. Let $\rho: \Gamma \rightarrow \operatorname{Isom}\left(\mathbb{H}^{n}\right)$ be a representation whose image is a non-elementary group. Then, for $k \geq 3$, there exists a smooth pseudodeveloping map $F: \mathbb{H}^{k} \rightarrow \mathbb{H}^{n}$ such that for all $x \in \mathbb{H}^{k}$,

$$
|\operatorname{Jac} F(x)| \leq 1
$$

moreover, equality holds at $x$ if and only if $d F_{x}: T_{x} \mathbb{H}^{k} \rightarrow T_{F(x)} \mathbb{H}^{n}$ is an isometry.

Assuming the image of $\rho$ is non-elementary, Lemma 3.1 now implies the inequality of Theorem 1.1. Indeed, by the definition of volume of a representation and the inequality in the lemma, it follows immediately that

$$
\operatorname{vol}(\rho) \leq \operatorname{vol}(F) \leq \operatorname{vol}(M)
$$

If the image of $\rho$ is elementary, then it is easy to check that $\operatorname{vol}(\rho)=0$. Thus, in either case, inequality (11) holds.

We now suppose that $\operatorname{vol}(\rho)=\operatorname{vol}(M)$ and proceed to show that the image of the pseudo-developing map $F$ is contained in a $k$-dimensional hyperbolic subspace of $\mathbb{H}^{n}$.

Since $\operatorname{vol}(\rho)=\operatorname{vol}(M)$, each of the inequalities of (2) is an equality. Hence, for each $x$ in $\mathbb{H}^{k}$, the inequality in (11) is equality. Thus the map $F$ is a Riemannian isometry.

We now recall some ideas and facts from Riemannian geometry, referring the reader to 9 for notation and details. We note that, in what follows, $C^{2}$-regularity of the pseudo-developing map is enough. 
Let $X$ denote a Riemannian manifold. A submanifold $N$ of $X$ is called minimal if it is a critical point of the volume function. A submanifold is locally minimal if, for each point $x$ of $N$, there exists a neighborhood $A$ of $x$ such that all perturbations of $N$ with support in $A$ do not decrease the volume of $N$. A submanifold $N$ of $X$ is totally geodesic if for any two points $x$ and $y$ in $N$, the geodesic joining $x$ and $y$ in $X$ is contained in $N$. We denote by $R^{X}$ and $\nabla^{X}$ (resp., $R^{N}$ and $\nabla^{N}$ ) the curvature tensor and the connection of $X$ (resp., $N)$.

For any two vector fields $U$ and $V$ in $N$, we denote by $\Pi(U, V)$ the second fundamental form of the submanifold $N$. Equivalently, if $\left\{\nu_{1} \ldots, \nu_{r}\right\}$ denotes an orthonormal frame of the orthogonal complement of $T N$ in $T X$, and if $l_{i}(U, V)$ denotes the real-valued fundamental form corresponding to $\nu_{i}$, then

$$
\nabla_{U}^{X} V-\nabla_{U}^{N} V=-\sum_{i=1}^{r} l_{i}(U, V) \nu_{i}=\Pi(U, V) .
$$

The strategy is to prove that the image of the map $F$ is a minimal submanifold of $\mathbb{H}^{n}$, and from this conclude that the image of $F$ is contained in a $k$-dimensional subspace of $\mathbb{H}^{n}$. To do this, we need the following standard results (9, Chapter V]).

Lemma 3.2. Let $N$ be a submanifold of a Riemannian manifold $X$. Then

1. $N$ is minimal if and only if the traces of all the real-valued second fundamental forms vanish (see [9, p.228]);

2. $N$ is totally geodesic if and only if the second fundamental form vanishes (see [9, p. 220]).

Lemma 3.3. The image $F\left(\mathbb{H}^{k}\right)$ of the map $F$ is contained in a locally minimal submanifold of $\mathbb{H}^{n}$.

Proof. Suppose not. Then by a perturbation of $F$ in a small ball $B$ of $\mathbb{H}^{k}$, we can decrease the volume of $F$. Indeed, by $\rho$-equivariantly perturbing $F$ in the $\Gamma$-orbit of $B$, we can find a pseudo-developing map $F^{\prime}: \mathbb{H}^{k} \rightarrow \mathbb{H}^{n}$ with a strictly smaller volume than that of $F$. But then $\operatorname{vol}(M)=\operatorname{vol}(\rho) \leq$ $\operatorname{vol}\left(F^{\prime}\right)<\operatorname{vol}(F)=\operatorname{vol}(M)$, a contradiction.

Lemma 3.4. Let $N$ be a locally minimal $k$-submanifold of a Riemannian $(k+r)$-manifold $X$. If, for all vector fields $U, V, W$, and $T$ we have

$$
R^{N}(U, V, W, T)=R^{X}(U, V, W, T),
$$


then $N$ is totally geodesic.

Proof. By (2) of Lemma 3.2, it suffices to show that the second fundamental form of $N$ vanishes. We again let $\left\{\nu_{1}, \ldots, \nu_{r}\right\}$ be an orthonormal frame of the orthogonal complement $T N$ of $T X$, and for each index $i$, we let $l_{i}(\cdot, \cdot)$ denote the real-valued fundamental form corresponding to $\nu_{i}$. By Gauss's theorem (see for example [9, Chapter V]), we conclude that for any point $p \in N$ and for any $u, v, w$, and $t$ in $T_{p} N$,

$$
R^{N}(u, v, w, t)=R^{X}(u, v, w, t)+\sum_{i=1}^{r}\left(l_{i}(u, w) l_{i}(v, t)-l_{i}(u, t) l_{i}(v, w)\right) .
$$

It then follows that for any $u, v, w$, and $t$ in $T_{p} N$,

$$
\left.\sum_{i=1}^{r}\left(l_{i}(u, w) l_{i}(v, t)-l_{i}(u, t) l_{i}(v, w)\right)\right)=0 .
$$

By hypothesis, $N$ is a locally minimal submanifold; therefore, by (11) of Lemma 3.2, we have that $\operatorname{tr}\left(l_{i}\right)=0$ for each $i=1, \ldots, r$.

Now let $e_{1}, \ldots, e_{k}$ denote an orthonormal basis of $T_{p} N$. Setting $u=t=e_{j}$ in the above equality, we have

$$
\left.\sum_{i=1}^{r}\left(l_{i}\left(e_{j}, w\right) l_{i}\left(v, e_{j}\right)-l_{i}\left(e_{j}, e_{j}\right) l_{i}(v, w)\right)\right)=0 .
$$

Setting $w=v$ and summing over the index $j$, we get

$$
\left.\sum_{j=1}^{k} \sum_{i=1}^{r} l_{i}^{2}\left(e_{j}, w\right)-\sum_{i=1}^{r} \operatorname{tr}\left(l_{i}\right) l_{i}(w, w)\right)=0
$$

Whence, by the vanishing trace condition of Lemma 3.2. we conclude that for any $p$ in $N$ and $w$ in $T_{p} N$,

$$
\sum_{i, j} l_{i}^{2}\left(e_{j}, w\right)=0
$$

It now follows that $l_{i}\left(e_{j}, w\right)=0$ for any $i, j$, and $w$, and hence that $l_{i} \equiv 0$ for $1 \leq i \leq r$. This shows that the second fundamental form vanishes at each point $p$ in $N$, which completes the proof of the lemma. 
We now apply Lemma 3.4 with $N=F\left(\mathbb{H}^{k}\right)$ and $X=\mathbb{H}^{n}$. Since $F$ is a Riemannian isometry, the hypothesis that $R^{N}=R^{X}$ is satisfied. By Lemma 3.3. $N$ is a locally minimal submanifold of $X$. Hence by Lemma 3.4. $N$ is totally geodesic. Therefore the map $F$ is an isometry from $\mathbb{H}^{k}$ to a $k$ dimensional subspace $H$ of $\mathbb{H}^{n}$, and it follows that the image of $\rho$ is contained in the group of isometries of $H$, as desired.

We claim now that $F: \mathbb{H}^{k} \rightarrow H$ is a covering map. Indeed, note that there exists an $r>0$ such that for any $x \in \mathbb{H}^{k}$, the restriction map $\left.F\right|_{B(x, r)}$ is an isometry onto its image. This easily implies the claim.

Since $\mathbb{H}^{k}$ is simply connected, the covering $F: \mathbb{H}^{k} \rightarrow H$ is a homeomorphism. Thus $F$ is a $\rho$-equivariant global isometry of $\mathbb{H}^{k}$. It follows that the representation $\rho$ is discrete and faithful.

Finally, we suppose that $\rho$ is a discrete and faithful representation into the group of isometries of a $k$-dimensional subspace $H$ of $\mathbb{H}^{n}$, and show that $\operatorname{vol}(\rho)=\operatorname{vol}(M)$. First, note that it is not restrictive to consider only those pseudo-developing maps for $\rho$ whose images are contained in $H$. Thus, after identifying $H$ with $\mathbb{H}^{k}$, we may assume that $n=k$.

Let $N=\mathbb{H}^{k} / \rho(\Gamma)$. By Mostow rigidity, the hyperbolic $k$-manifolds $M$ and $N$ are isometric, and in particular, $\operatorname{vol}(N)=\operatorname{vol}(M)$. Now let $D$ be any pseudo-developing map for $\rho$. Since $D$ is $\rho$-equivariant, it induces a map $g: M \rightarrow N$, and by definition, $\operatorname{vol}(D)=\int_{M}\left|g^{*} \omega\right|$, where $\omega$ is the hyperbolic volume form of $N$. Hence

$$
\operatorname{vol}(N)=\operatorname{vol}(M)=\left|\int_{M} g^{*} \omega\right| \leq \int_{M}\left|g^{*} \omega\right|=\operatorname{vol}(D) .
$$

It follows that

$$
\operatorname{vol}(M) \leq \operatorname{vol}(\rho)=\inf _{D}\{\operatorname{vol}(D)\}
$$

and we have already shown (see inequality (2) after Lemma 3.1) that the reverse inequality also holds. This completes the proof of Theorem 1.1] when $M$ is a compact manifold.

\section{The finite-volume case}

In this section we complete the proof of Theorem 1.1 proving the result in the finite-volume case. The main difference from the previous case is that, as our manifolds are no longer compact, we need to work with proper maps; since we work at the level of universal coverings, we need an equivariant notion 
of properness. We keep here all the notation and definitions of previous sections, except that in the sequel $M$ will denote an oriented, complete, noncompact, hyperbolic $k$-manifold of finite volume with $k \geq 3$. We will also need to modify the definition of the volume of a representation.

The manifold $M$ is diffeomorphic to the interior of a compact manifold $\bar{M}$ whose boundary consists of Euclidean $(k-1)$-manifolds. (See, for example, [1]). In particular, for each boundary component $T \subset \partial \bar{M}$ the group $\pi_{1}(T)<\pi_{1}(M)=\Gamma<\operatorname{Isom}\left(\mathbb{H}^{\mathrm{k}}\right)$ is an abelian parabolic group. The following lemma is easy to check.

Lemma 4.1. Let $G$ be an abelian group of isometries of a hyperbolic space $\mathbb{H}^{m}$. Then the set $\operatorname{Fix}(G) \subset \overline{\mathbb{H}^{\mathrm{m}}}$ of points which are fixed by $G$ is non-empty.

We note that $G$ may have no fixed point in $\partial \mathbb{H}^{m}$ (for example if $G<$ Isom $\left(\mathbb{H}^{3}\right)$ is the dihedral group generated by two rotations of angle $\pi$ around orthogonal axes).

Up to conjugacy, a peripheral subgroup of $\pi_{1}(M)$ has a unique fixed point, which lies in $\partial \mathbb{H}^{k}$. Thus, for each $T \subset \partial \bar{M}$, each conjugate of $\pi_{1}(T)$ in $\pi_{1}(M) \subset \operatorname{Isom}\left(\mathbb{H}^{\mathrm{k}}\right)$ corresponds to its fixed point in $\partial \mathbb{H}^{k}$.

We can now give the definition of a properly-ending map.

Definition 4.2 (Properly ending maps). Let $\rho: \pi_{1}(M) \rightarrow \operatorname{Isom}\left(\mathbb{H}^{n}\right)$ be a representation, and let $D: \mathbb{H}^{k} \rightarrow \mathbb{H}^{n}$ be a $\rho$-equivariant map. We say that $D$ properly ends if for each $T \subset \partial \bar{M}$, if $\xi=\operatorname{Fix}\left(\pi_{1}(\mathrm{~T})\right)$ and $\alpha(t)$ is a geodesic ray ending at $\xi$, then all limit points of $D(\alpha(t))$ lie either in $\operatorname{Fix}\left(\rho\left(\pi_{1}(\mathrm{~T})\right)\right) \subset \overline{\mathbb{H}^{\mathrm{n}}}$ or in a finite union of $\rho\left(\pi_{1}(T)\right)$-invariant geodesics.

Definition 4.3 (Volume of a representation). The volume $\operatorname{vol}(\rho)$ of a representation $\rho$ is defined by

$$
\operatorname{vol}(\rho)=\inf _{D}\{\operatorname{vol}(D)\},
$$

where the infimum is taken over the set of all properly-ending pseudo-developing maps $D$ for $\rho$.

Remark 4.4. It is easy to construct properly-ending pseudo-developing maps. (See [6, 8].) We need to work with such maps because otherwise, one can construct (non-properly-ending) pseudo-developing maps with volume zero. (For example, one can collapse $M$ to any of its spines.) Also, we note that the above definition of volume "extends" the previous one given for compact manifolds. Indeed, if $M$ is compact, then any pseudo-developing map properly ends. 
We now need to recall the definition and properties of the barycenter of measures in $\overline{\mathbb{H}^{n}}$, referring to [4, 7] for details. (The reader who is familiar with such constructions may skip directly to Lemma 4.5.) Let $\beta$ be a probability Borel measure on $\partial \mathbb{H}^{n}$. We define a function $\mathcal{B}_{\beta}: \mathbb{H}^{n} \rightarrow \mathbb{R}$ by

$$
\mathcal{B}_{\beta}(y)=\int_{\partial \mathbb{H}^{n}} B(y, \theta) d \beta(\theta)
$$

where $B(y, \theta)$ is the Busemann function of $\mathbb{H}^{n}$. Then we have

1. If $\beta$ is not concentrated in two points, then $\mathcal{B}_{\beta}$ is strictly convex (because its Hessian is the $\beta$-average of the Hessians of the Busemann functions $B(y, \cdot))$ and goes to $\infty$ as $y$ goes to $\partial \mathbb{H}^{n}$.

2. If $\beta$ is not the sum of two Dirac delta measures with the same weight, then $\mathcal{B}_{\beta}$ has a unique minimum (possibly $-\infty$ ) in $\overline{\mathbb{H}^{n}}$. Such a minimum is attained in $\partial \mathbb{H}^{n}$ if and only if $\beta$ has an atom of weight greater that $\frac{1}{2}$. The point $\operatorname{bar}(\beta)$ where $\mathcal{B}_{\beta}$ attains its minimum is called the barycenter of $\beta$.

3. If $\beta$ is the sum $\frac{1}{2}\left(\delta_{\theta_{1}}+\delta_{\theta_{2}}\right)$ of two Dirac delta measures concentrated in $\theta_{1}$ and $\theta_{2}$, then $\mathcal{B}_{\beta}$ is convex and constant on the geodesic joining $\theta_{1}$ and $\theta_{2}$, where it attains its minimum.

4. If $\beta$ is a probability measure on $\mathbb{H}^{n}$, its barycenter is defined by taking the convolution with the family of visual measures as follows. Let $\nu_{O^{\prime}}$ be the standard probability measure on $\partial \mathbb{H}^{n} \simeq \mathbb{S}^{n-1}$ in the disc model with center $O^{\prime}$. For every $y \in \mathbb{H}^{n}$, define $\nu_{y}=\psi_{*} \nu_{O^{\prime}}$, where $\psi$ is any isometry mapping $O^{\prime}$ to $y$. (Note that this is well-defined because $\nu_{O^{\prime}}$ is $\operatorname{Stab}\left(O^{\prime}\right)$-invariant.) Now define $\bar{\beta}$, a probability measure on $\partial \mathbb{H}^{n}$, by

$$
\int_{\partial \mathbb{H}^{n}} \varphi(\theta) d \bar{\beta}(\theta)=\int_{\mathbb{H}^{n}}\left(\int_{\partial \mathbb{H}^{n}} \varphi(\theta) d \nu_{y}(\theta)\right) d \beta(y) .
$$

The barycenter of $\beta$ is defined as the barycenter of $\bar{\beta}$.

5. The barycenter is defined in the same way for non-negative measures of finite, non-zero mass. For any positive constant $c$, we have $\operatorname{bar}(c \beta)=$ $\operatorname{bar}(\beta)$. 
6. The barycenter is continuous w.r.t. the weak-* convergence of measures, that is, if $\left\{\beta_{i}\right\}$ is a sequence of measures with barycenter and converging to a measure $\beta$ with barycenter, then $\left\{\operatorname{bar}\left(\beta_{i}\right)\right\} \rightarrow \operatorname{bar}(\beta)$.

7. The barycenter is equivariant by isometries, that is, $\operatorname{bar}\left(\gamma_{*} \beta\right)=\gamma(\operatorname{bar}(\beta))$ for any isometry $\gamma$ (where $\gamma_{*} \beta$ denotes the push-forward via $\gamma$ of the measure $\beta$ ).

What we need to complete the proof of Theorem [1.1] is the following fact. (Compare with Lemma 3.1.)

Lemma 4.5. For any $\varepsilon>0$ and for any non-elementary representation $\rho$, there exists a map $F^{\varepsilon}: \mathbb{H}^{k} \rightarrow \mathbb{H}^{n}$ such that

1. The map $F^{\varepsilon}$ is smooth and $\rho$-equivariant.

2. $\left|\operatorname{Jac} F^{\varepsilon}(x)\right| \leq 1+\varepsilon$, and equality holds if and only if $d F_{x}^{\varepsilon}: T_{x} \mathbb{H}^{k} \rightarrow$ $T_{F^{\varepsilon}(x)} \mathbb{H}^{n}$ is a homothety.

3. $\lim _{\varepsilon \rightarrow 0} F^{\varepsilon}=F$, where $F$ is the map of Lemma 3.1.

4. The map $F^{\varepsilon}$ properly ends.

Before proving Lemma 4.5, we show how it implies Theorem 1.1. The inequality directly follows from points (11), (2) and (41). If $\operatorname{vol}(\rho)=\operatorname{vol}(M)$, then by point (3) one gets that $\operatorname{vol}(F)=\operatorname{vol}(M)$. (Note that, a priori, the map $F$ of Lemma 3.1 does not end properly). The proof now follows exactly as in the compact case.

Proof of Lemma 4.5. The maps $F^{\varepsilon}$ are the so called $\varepsilon$-natural maps introduced by Besson, Courtois, and Gallot. We begin by recalling their construction. We omit most details, referring to 4, 7, 2, 3, for a complete discussion on the construction of natural maps.

For any $\varepsilon>0$, we set

$$
s=(k-1)(1+\varepsilon) \text {. }
$$

Let $O$ be a marked point in $\mathbb{H}^{k}$, and let $c(s)=\sum_{\gamma \in \Gamma} e^{-s d(O, \gamma O)}$. It turns out that $c(s)<\infty$, for any $s>k-1$. 
For any $x \in \mathbb{H}^{k}$, we define $\mu_{x}^{\varepsilon}$ a positive Borel measure on $\mathbb{H}^{k}$ by

$$
\mu_{x}^{\varepsilon}=\frac{1}{c(s)} \sum_{\gamma \in \Gamma} e^{-s d(x, \gamma O)} \delta_{\gamma O},
$$

where $\delta_{\gamma O}$ denotes the Dirac measure concentrated on the point $\gamma O$.

Next, we define the measures $\eta_{x}^{\varepsilon}$ on $\mathbb{H}^{n}$ and $\lambda_{x}^{\varepsilon}$ on $\partial \mathbb{H}^{n}$, respectively, as the equivariant push-forward of $\mu_{x}^{\varepsilon}$ and its convolution with the family $\left\{\nu_{y}\right\}$ of visual measures. Namely, choose a point $O^{\prime} \in \mathbb{H}^{n}$ and define

$$
\eta_{x}^{\varepsilon}=\frac{1}{c(s)} \sum_{\gamma \in \Gamma} e^{-s d(x, \gamma O)} \delta_{\rho(\gamma) O^{\prime}} \quad \text { and } \quad \lambda_{x}^{\varepsilon}=\frac{1}{c(s)} \sum_{\gamma \in \Gamma} e^{-s d(x, \gamma O)} \nu_{\rho(\gamma) O^{\prime}} .
$$

The map $F^{\varepsilon}$ is defined by

$$
F^{\varepsilon}(x)=\operatorname{bar}\left(\eta_{x}^{\varepsilon}\right)=\operatorname{bar}\left(\lambda_{x}^{\varepsilon}\right)=\operatorname{bar}\left(\frac{\lambda_{x}^{\varepsilon}}{\left\|\lambda_{x}^{\varepsilon}\right\|}\right) .
$$

Under our present hypotheses we have the following:

- (Besson, Courtois, Gallot [4, Théorème 1.10]) The map $F^{\varepsilon}$ satisfies conditions (11) and (2) of Lemma 4.5.

- (Francaviglia [7, Proposition 1.5]) The maps $F^{\varepsilon}$ satisfy condition (3) of Lemma 4.5.

Therefore, it remains only to prove that for each $\varepsilon>0$, the map $F^{\varepsilon}$ properly ends. Let $T \subset \bar{M}$ be a boundary component and let $\pi_{1}(T)$ be (one of) the corresponding parabolic subgroups of $\pi_{1}(M)$, and let $\xi=\operatorname{Fix}\left(\pi_{1}(T)\right)$.

The idea is now the following. For $x \in \mathbb{H}^{k}$, we have

$$
\eta_{x}^{\varepsilon}=\frac{e^{-s d(x, O)}}{c(s)} \sum_{\gamma \in \Gamma} e^{-s(d(x, \gamma O)-d(x, O))} \delta_{\rho(\gamma) O^{\prime}},
$$

and by point (15) of page 9 we have

$$
F^{\varepsilon}(x)=\operatorname{bar}\left(\eta_{x}^{\varepsilon}\right)=\operatorname{bar}\left(\frac{c(s)}{e^{-s d(x, O)}} \lambda_{x}^{\varepsilon}\right)=\operatorname{bar}\left(\sum_{\gamma \in \Gamma} e^{-s(d(x, \gamma O)-d(x, O))} \nu_{\rho(\gamma) O^{\prime}}\right) .
$$


Now, let $\alpha(t)$ be a geodesic ray ending at $\xi$. As $t \rightarrow \infty$, we have

$$
\sum_{\gamma \in \Gamma} e^{-s(d(\alpha(t), \gamma O)-d(\alpha(t), O))} \nu_{\rho(\gamma) O^{\prime}} \stackrel{*}{\rightarrow} \sum_{\gamma \in \Gamma} e^{-s B(\xi, \gamma O)} \nu_{\rho(\gamma) O^{\prime}},
$$

where $B(\cdot, \cdot)$ denotes the Busemann function normalized at $O$. Thus, from point (6) of page 10] we would get that, as $t \rightarrow \infty$

$$
F^{\varepsilon}(\alpha(t)) \rightarrow \operatorname{bar}\left(\sum_{\gamma \in \Gamma} e^{-s B(\xi, \gamma O)} \nu_{\rho(\gamma) O^{\prime}}\right)
$$

which one might expect should be fixed by the elements of $\rho\left(\pi_{1}(T)\right)$, because the limit measure $\sum_{\gamma \in \Gamma} e^{-s B(\xi, \gamma O)} \nu_{\rho(\gamma) O^{\prime}}$ is $\rho\left(\pi_{1}(T)\right)$-invariant.

Unfortunately, the limit measure $\sum_{\gamma \in \Gamma} e^{-s B(\xi, \gamma O)} \nu_{\rho(\gamma) O^{\prime}}$ has no finite mass, whence its barycenter is not defined.

In order to overcome this difficulty, some more work is required. For each $x$ the measure $\lambda_{x}^{\varepsilon} /\left\|\lambda_{x}^{\varepsilon}\right\|$ is a probability measure on $\partial \mathbb{H}^{n} \simeq \mathbb{S}^{n-1}$. Since $\mathbb{S}^{n-1}$ is compact, the set of probability measures on $\partial \mathbb{H}^{n}$ is weak-* compact. Therefore, after possibly passing to a subsequence as $x \rightarrow \xi$ along the ray $\alpha$, the measures $\lambda_{x}^{\varepsilon} /\left\|\lambda_{x}^{\varepsilon}\right\|$ converge to a probability measure $\lambda_{\xi}$ on $\partial \mathbb{H}^{n}$. (The measure $\lambda_{\xi}$ depends on the chosen subsequence).

We show now that $\lambda_{\xi}$ is $\rho\left(\pi_{1}(T)\right)$-invariant. Let $\psi \in \pi_{1}(T)<\pi_{1}(M)=$ $\Gamma<\operatorname{Isom}\left(\mathbb{H}^{k}\right)$. Since

$$
\rho(\psi)_{*} \lambda_{x}^{\varepsilon}=\frac{1}{c(s)} \sum_{\gamma \in \Gamma} e^{-s d(x, \gamma O)} \nu_{\rho(\psi \gamma) O^{\prime}}=\frac{1}{c(s)} \sum_{\gamma \in \Gamma} e^{-s d\left(x, \psi^{-1} \gamma O\right)} \nu_{\rho(\gamma) O^{\prime}}
$$

we have

$$
\rho(\psi)_{*} \lambda_{x}^{\varepsilon}-\lambda_{x}^{\varepsilon}=\frac{1}{c(s)} \sum_{\gamma \in \Gamma} e^{-s d(x, \gamma O)}\left(e^{-s\left(d\left(x, \psi^{-1} \gamma O\right)-d(x, \gamma O)\right)}-1\right) \nu_{\rho(\psi \gamma) O^{\prime}} .
$$

Using the hyperbolic law of sines on the triangles with vertices $x, \gamma O$ and $\psi^{-1} \gamma O$, one sees that there exists a function $E(x)$ such that $E(x) \rightarrow 0$ as $x \rightarrow \xi$ and

$$
\left|e^{-s\left(d\left(x, \psi^{-1} \gamma O\right)-d(x, \gamma O)\right)}-1\right|<E(x),
$$

whence

$$
\left\|\rho(\psi)_{*} \lambda_{x}^{\varepsilon}-\lambda_{x}^{\varepsilon}\right\|<E(x)\left\|\lambda_{x}^{\varepsilon}\right\|
$$


Since $\left\|\lambda_{x}^{\varepsilon}\right\|=\left\|\rho(\psi)_{*} \lambda_{x}^{\varepsilon}\right\|$, we have that $\lambda_{x}^{\varepsilon} /\left\|\lambda_{x}^{\varepsilon}\right\|$ and $\rho(\psi)_{*} \lambda_{x}^{\varepsilon} /\left\|\rho(\psi)_{*} \lambda_{x}^{\varepsilon}\right\|$ have the same limit $\lambda_{\xi}$. It follows that $\lambda_{\xi}$ is $\rho\left(\pi_{1}(T)\right)$-invariant.

Now we have two cases: either $\lambda_{\xi}=\frac{\delta_{\theta_{1}}+\delta_{\theta_{2}}}{2}$, or not. In the latter case, by point (6) of page 10.

$$
F^{\varepsilon}(x) \rightarrow \operatorname{bar}\left(\lambda_{\xi}\right),
$$

which, by point (7) of page 10, is fixed by the elements of $\rho\left(\pi_{1}(T)\right)$.

In the former case, the barycenter of $\lambda_{\xi}$ is not defined. Nevertheless, one can show that the functions $\mathcal{B}_{\lambda_{x}^{\varepsilon}}(y)$, defined at page 9, converge to $\mathcal{B}_{\lambda_{\xi}}(y)$. Since, for each $\varepsilon, \operatorname{bar}\left(\lambda_{x}^{\varepsilon}\right)$ is the point where $\mathcal{B}_{\lambda_{x}^{\varepsilon}}$ takes its minimum, they converge to a minimum of $\mathcal{B}_{\lambda_{\xi}}$ that, by point (3) of page 9 lies in the geodesic joining $\theta_{1}$ and $\theta_{2}$. Such geodesic is $\rho\left(\pi_{1}(T)\right.$ )-invariant because the invariance of $\lambda_{\xi}$. This completes the proof of Lemma 4.5, and hence the proof of Theorem 1.1.

\section{References}

[1] Riccardo Benedetti and Carlo Petronio. Lectures on hyperbolic geometry. Universitext. Springer-Verlag, Berlin, 1992.

[2] Gérard Besson, Gilles Courtois, and Sylvestre Gallot. Entropies et rigidités des espaces localement symétriques de courbure strictement négative. Geom. Funct. Anal., 5(5):731-799, 1995.

[3] Gérard Besson, Gilles Courtois, and Sylvestre Gallot. Minimal entropy and Mostow's rigidity theorems. Ergodic Theory Dynam. Systems, 16(4):623-649, 1996.

[4] Gérard Besson, Gilles Courtois, and Sylvestre Gallot. Lemme de Schwarz réel et applications géométriques. Acta Math., 183(2):145-169, 1999.

[5] Gérard Besson, Gilles Courtois, and Sylvestre Gallot. Inégalité de milnor-wood géométriques. 2004. In preparation.

[6] Nathan M. Dunfield. Cyclic surgery, degrees of maps of character curves, and volume rigidity for hyperbolic manifolds. Invent. Math., 136(3):623$657,1999$. 
[7] Stefano Francaviglia. Constructing equivariant maps for representations. Preprint DMA, available version arXiv:math.GT/0405028.

[8] Stefano Francaviglia. Hyperbolic volume of representations of fundamental groups of cusped 3-manifolds. Int. Math. Res. Not., (9):425-459, 2004.

[9] Sylvestre Gallot, Dominique Hulin, and Jacques Lafontaine. Riemannian geometry. Universitext. Springer-Verlag, Berlin, second edition, 1990.

[10] William M. Goldman. Characteristic classes and representations of discrete subgroups of Lie groups. Bull. Amer. Math. Soc. (N.S.), 6(1):9194, 1982 .

[11] B. Klaff. Boundary slopes of knots in closed 3-manifolds with cyclic fundamental group. PhD thesis, University Illinois-Chicago, 2003.

[12] W. P. Thurston. The geometry and topology of 3-manifolds. Mimeographed notes. Princeton University Mathematics Department, 1979 . 